\title{
A 75-year-old man with new onset dyspnea and haemoptysis
}

\begin{abstract}
Foreign body aspiration (FBA) is a common life-threatening condition which mostly occurs in children and elderly due to lack of airway protection mechanisms. A 75-year-old man was admitted to our outpatient clinic with complaints of a permanent shortness of breath for approximately 2 months and haemoptysis for two weeks. He had prescribed bronchodilators and levofloxacin with a diagnosis of COPD exacerbation but he didn't experience any relief though he used the medication regularly. He was a water-pipe smoker for 50 years. He reported to swallow 3 olive seeds in a day, after he watched an herbalist which had advised to do so, on a TV program. On presentation, wheezing was heard over the right hemi thorax and crackles were noted at the left base. His chest radiograph showed a suspicious heterogenic density behind the left heart silhouette. Bronchoscopy showed a polypoid lesion on the posterior wall of the left main bronchus, whereas an oval shaped mobile body (olive seed) was observed over the right main bronchus, under the right upper lobe division. The pathological diagnosis of the specimen taken from the polypoid lesion was compatible with active chronic granulation. We removed the olive seed by grasping forceps and the symptoms of the patient dissolved as well as the radiological findings. This is a demonstrative case reminding to consider FBA in elderly with sub acute respiratory symptoms.
\end{abstract}

Keywords: foreign body aspiration, bronchoscopy, chronic cough
Volume I Issue 3 - 2014

\section{Eyup Sabri Ucan,' Can Sevinc,' Aylin Ozgen Alpaydin,' Kemal Can Tertemiz,' Pervin Hanci $^{2}$ \\ 'Department of Chest Disease, Dokuz Eylul University, Turkey ${ }^{2}$ Kastamonu Public Hospital,Turkey}

Correspondence: Kemal Can Tertemiz, Dokuz Eylul University Faculty of Medicine, Department of Chest Disease, Dokuz Eylul Universitesi Hastanesi Inciralti/lzmir, Turkey, Tel +902324I 23809 , Email tkemalcan@yahoo.com

Received: September 26, 2014 | Published: September 30, 2014
Abbreviations: FBA, foreign body aspiration; CT, computed tomography; FB, flexible bronchoscopes

\section{Introduction}

A 75-year-old man was admitted to our outpatient clinic with complaints of shortness of breath and haemoptysis. He defined no respiratory symptoms 2 months to the admission. He could not remember the how or when the dyspnea began, however he reported a permanent shortness of breath for approximately two months, without association of a specific condition. Two weeks before, he experienced blood in his sputum when he coughed and this symptom was repeated two or three times a day. His primary care physician prescribed him bronchodilators and levofloxacin with the diagnosis of chronic obstructive pulmonary diseases and pneumonia. He did not experience any relief though he used the medication regularly. His remaining medical history was not significant for any chronic diseases. His medications included irregularly used omeprazole and pain killers. He was a water-pipe smoker for 50years. He reported to swallow 3 olive seeds in a day, after he watched a herbalist which had advised to do so, on a television program.

\section{Physical examination}

On presentation, he was a febrile with a heart rate of 82 beats $/ \mathrm{min}$ and a blood pressure of $110 / 72 \mathrm{~mm} \mathrm{Hg}$. However, he was dyspneic and his pulse oximetric saturation was $96 \%$ while breathing room air Wheezing was heard over the right hemi thorax and crackles were noted at the left base. Other system examinations were normal.

\section{Laboratory test and imaging}

Blood tests done showed a mild leukocytosis $(17300 \mathrm{IU} / \mathrm{mL})$; renal and hepatic panels were normal. Arterial blood gas analyses were also normal. His chest radiograph showed no abnormality, except a suspicious heterogenic density behind the left heart silhouette. CT scans of the thorax are shown below in Figure 1A and Figure 1B. Bronchoscopy was then performed, which showed a polyploidy lesion on the posterior wall of the left main bronchus, whereas an oval shaped mobile body was observed over the right main bronchus, under the right upper lobe division (Figure 2A) (Figure 2B). The pathological diagnosis of the specimen taken from the polypoid lesion was compatible with active chronic granulation.

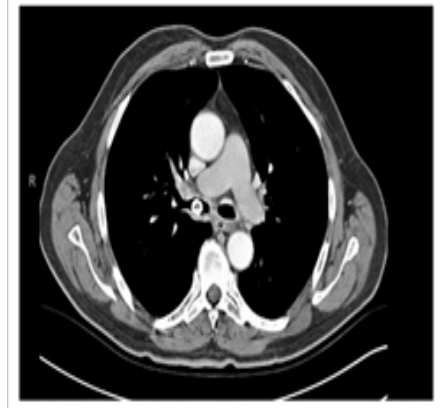

(A)

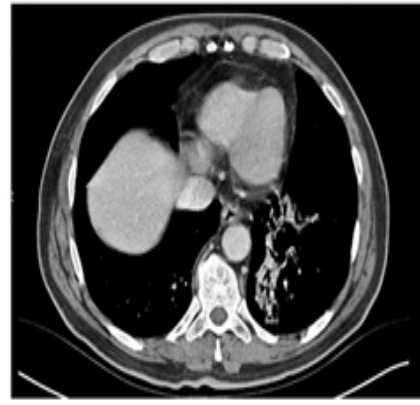

(B)
Figure I A) CT scans of the case demonstrating a foreign body in the right main bronchus. B) Consolidation in left lower lobe. 

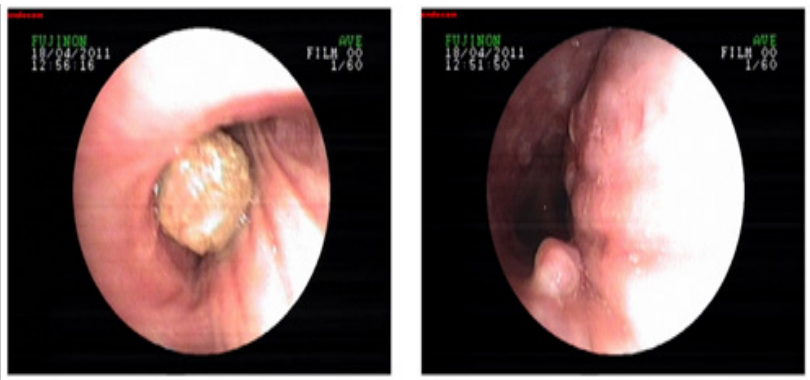

(A)

(B)

Figure 2 A) Bronchoscopic images showing an oval shaped body in the right main bronchus, under the right upper lobe division. B) A polypoid lesion on the posterior wall of the left main bronchus.

\section{What is the diagnosis?}

Diagnosis: Foreign body "Olive seed" aspiration and granulomatous reaction due to chronic inflammation.

\section{Discussion}

Foreign body aspiration (FBA) is a common medical condition both in children and adults which demands prompt recognition and action, since it is associated with life-threatening complications. Nearly $80 \%$ of FBAs occur in children younger than 15 years old. ${ }^{1}$ However, the prevalence of FBA in adults increase with age as the airway protective mechanisms is impaired. ${ }^{2}$ Dysphagia, dementia, cerebrovascular disease, sedative or hypnotic drug use that alter the swallowing function and consciousness have been defined as predisposing factors associated with aspiration syndromes in elderly. However, other factors causing unconsciousness like alcohol intoxication, trauma, epilepsy and general anaesthesia may also facilitate FBA. ${ }^{3}$

Clinical presentation of FBA may differ from life-threatening airway obstruction to nonspecific symptoms according to the level of obstruction and the features of the material aspirated. Organic materials develop a severe mucosal reaction and granulation tissue leading to polyp formation, on the other hand inorganic bodies may remain silent for manyyears. ${ }^{4}$ The most common symptoms for FBA have been reported as sudden onset of dyspnea, cough, choking, and vomiting. ${ }^{5}$ In adults, typical presentation is not common and foreign body may stay undetected for a long time. Delayed diagnosis may lead to development of recurrent haemoptysis, persistent cough, recurrent pneumonia and airway obstruction with irreversible lung injury Nonspecific respiratory symptoms are usually thought to be related with other medical conditions and especially older patients are diagnosed with other respiratory diseases such as chronic obstructive pulmonary disease. ${ }^{6}$

The most important step in the diagnostic approach of FBA is clinical suspicion. Especially in children and elderly who present with common symptoms of FBA the diagnostic workup should include the procedures for FBA. Standard chest radiography and lateral graphs are the first examinations in the evaluation of FBA, although most of the aspirated subjects are organic and radiolucent. ${ }^{7}$ Eight to $80 \%$ of the chest radiographies may be negative for FBA. Radiological findings reported to be associated with FBA are bronchopneumonia, Atelectasis, one sided emphysema, lung abscess, empyema, bronchiectasis and mediastinal shift. ${ }^{2,8}$ Thorax computed tomography (CT) is not recommended in the first line evaluation of FBA; however it may detect aspirated materials in the lumen of tracheobronchial tree in adults with chronic respiratory symptoms. Atelectasis, unilateral hyperlucency, bronchiectasis, consolidation and tree-in-bud are some of the other findings related with FBA in thorax $\mathrm{CT} .{ }^{9}$

Since 1897, both rigid and flexible bronchoscopes (FB) have been used for the diagnosis and removal of foreign bodies. FB is recommended in the initial evaluation, while rigid bronchoscopy remains the standard therapeutic approach because of its better visualization and working area. FB has been reported with high success rates ranging from $61 \%$ to $90 \% .{ }^{8}, 10$ The procedure is generally safe and well tolerated, however due to dislocation of foreign body clinical deterioration may develop. Therefore, emergency equipment and conditions to continue with rigid bronchoscope should be achieved to the procedure (case1). In patients with craniofacial trauma and mechanical ventilation FB should be performed, whereas rigid bronchoscope is the preferred procedure in children. Foreign bodies often can be removed with FB under local anesthesia, and it should be the initial diagnostic and therapeutic technique for the removal of foreign bodies in adults. ${ }^{1}$ In a retrospective case-control analysis of safety and effectiveness of FB in the removal of foreign bodies in elderly $>75$ years, it was shown that FB was safe and $88 \%$ effective in the advanced elderly. ${ }^{6}$

Several different equipments have been designed for bronchoscopic procedure of foreign body removal. They are inserted through the bronchoscopic channel. Foreign body forceps's, snares, catheters are some of these. Cryotherapy is another option. Hypoxia, bleeding, laryngeal edema are some of the complications that might develop during the procedure. ${ }^{8}$

After extraction of the foreign body most of the clinical syndromes such as recurrent pneumonia or obstructive emphysema get cured. However, a significant tissue reaction develops in the delayed cases and in some cases like bronchiectasis complete resolution may not be achieved. ${ }^{1}$ Surgery should be performed as a final therapeutic approach when all the other techniques fail.

\section{Clinical course}

Our patient could not remember that he aspirated olive seed. He did not experience choking and vomiting. However, bronchoscopy revealed the foreign body, which we considered to Trans locate from left to right after the development of a granulation tissue due to chronic irritation. We removed the olive seed by grasping forceps and the symptoms of the patient dissolved.

\section{Clinical pearls}

i. FBA mostly occurs in children and elderly due to lack of airway protection mechanisms.

ii. Typical symptoms include sudden onset of dyspnea and choking, while there may be nonspecific respiratory symptoms are silent clinical course in the undiagnosed cases.

iii. Chest X ray is the initial diagnostic procedure, although only $20 \%$ of the aspirated materials are radio opaque.

iv. FB is the safe and diagnostic and therapeutic approach in adults and elderly.

v. Chronic respiratory symptoms in children and elderly should raise the suspicion of FBA. 


\section{Acknowledgements}

None.

\section{Conflict of interest}

The author declares no conflict of interest.

\section{References}

1. Chen $\mathrm{CH}$, Lai CL, Tsai TT, et al. Foreign body aspiration into the lower airways in Chinese adults. Chest. 1997;112(1):129-133.

2. Debeljak A, Sorli J, Music E, et al. Bronchoscopic removal of foreign bodies in adults: experience with 62 patients from 1974-1998. Eur Respir J. 1999;14(4):792-795.

3. Freiman MA, McMurray JS. Unique presentation of a bronchial foreign body in an asymptomatic child. Ann Otol Rhinol Laryngol. 2001;110(6):495-497.

4. Dikensoy O, Usalan C, Filiz A. Foreign body aspiration: clinical utility of flexible bronchoscopy. Postgrad Med J. 2002;78(921):399-403.
5. Willett LL, Barney J, Saylors G, et al. An unusual cause of chronic cough. Foreign body aspiration. J Gen Intern Med. 2006;21(2):C1-C3.

6. Boyd M, Watkins F, Singh S, et al. Prevalence of flexible bronchoscopic removal of foreign bodies in the advanced elderly. Age Ageing. 2009;38(4):396-400.

7. Boyd M, Chatterjee A, Chiles C, et al. Tracheobronchial foreign body aspiration in adults. South Med J. 2009;102(2):171-174.

8. Tang LF, Xu YC, Wang YS, et al. Airway foreign body removal by flexible bronchoscopy: experience with 1027 children during 2000-2008. World J Pediatr. 2009;5(3):191-195.

9. Mise K, Jurcev Savicevic A, Pavlov N, et al. Removal of tracheobronchial foreign bodies in adults using flexible bronchoscopy:experience 1995-2006. Surg Endosc. 2009;23(6):1360-1364.

10. Bai W, Zhou X, Gao X, et al. The value of chest CT in the diagnosis and management of tracheobronchial foreign bodies. Pediatr Int 2011;53(4):515-518. 